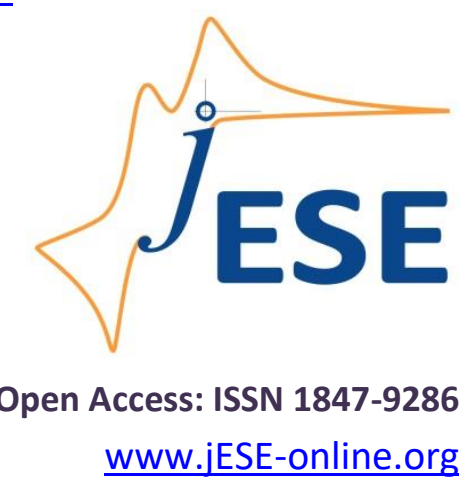

Review

\title{
Advances in understanding Li battery mechanisms using impedance spectroscopy
}

\author{
Jože Moškon ${ }^{1}$, Sara Drvarič Talian ${ }^{1}$, Robert Dominko ${ }^{1}$, Miran Gaberšček ${ }^{1,2, 凶}$ \\ ${ }^{1}$ Department of Materials Chemistry, National Institute of Chemistry, Hajdrihova 19, 1000 \\ Ljubljana, Slovenia \\ ${ }^{2}$ Faculty of Chemistry and Chemical Technology University of Ljubljana, Večna pot 113, 1000 \\ Ljubljana, Slovenia
}

Corresponding author: ${ }^{\bowtie}$ miran.gaberscek@ki.si; Tel.: +386-1-4760-320

Received: October 7, 2019; Revised: January 8, 2020; Accepted: January 8, 2020

\begin{abstract}
The use of impedance spectroscopy in the field of modern batteries is demonstrated on three systems: lithium ion batteries represented by lithium iron phosphate (LFP) and lithium cobalt oxide (LCO) electrodes, a porous carbon cathode in contact with polysulfides and a metallic lithium anode exhibiting dendritic growth. In all cases, systematic experiments are shown where the type and composition of electrochemical cell is varied in order to identify the main processes contributing to the impedance response. The experiments are upgraded with appropriate models, using mainly the transmission line approach. The approach allows establishment of clear correlations between the composition and morphology of electrodes on one hand and the measured impedance features on the other. As the transmission line models are based on the use of physically well-defined elements, the approach allows a quantitative description of the main processes (diffusion, reaction, migration across films and contacts) taking place in modern battery electrodes.
\end{abstract}

\section{Keywords}

Lithium ion batteries; lithium sulfur batteries; transmission line model; porous electrode model; dendrites diffusion; electrochemical reaction.

\section{Introduction}

Lithium batteries have been successfully marketed since 1991 when Sony introduced the first rechargeable Li battery based on lithium cobalt oxide as cathode and graphite as anode material. Later on, many other materials have been proposed both as a cathode or anode in order to further improve the battery performance. Despite the commercial success and continuous improvement of Li batteries, the mechanisms occurring in these devices have remained only partially explained. Whereas the development of new methodologies (microscopy, various spectroscopies etc.) has 
provided significant insight into local phenomena in certain electrode materials, evaluation of the overall electrochemical performance of given electrode still mainly relies on the use of conventional electrochemical methods and approaches. Among those, impedance spectroscopy is known to be the most accurate and also most powerful as regards the discrimination of various electrochemical steps occurring within the electrochemical cell.

This short review paper demonstrates how impedance spectroscopy can help to improve the understanding of main mechanisms taking place in typical lithium battery cells. For the sake of some generality, we present typical results obtained on three significantly different electrode types: (i) insertion cathodes, (ii) sulfur cathodes having different morphologies and (iii) a metallic lithium anode. Most of the results presented here are related to our own past experiments and modeling, occasionally other results are also referred to and commented on.

\section{Results and discussion}

\section{Insertion electrodes}

Insertion electrodes are still the most popular type of Li battery electrodes [1,2]. They predominantly consist of active particles of different shapes with the sizes between $c a .10 \mathrm{~nm}$ to several micrometers. The active particles are bound together by a polymeric binder so that a porous matrix is formed. During the cell manufacture, the pores are filled with electrolyte that contains ions of active species. Some carbon black or a similar electronic conductor is also added in order to ensure sufficient electronic conductivity of the whole electrode matrix. The electrode composite is pressed on a current collector which mainly consists of copper (for anodes) or aluminium (for cathodes). The main transport-reaction steps taking place in insertion electrodes are schematically shown in Figure 1a (for clarity the binder and conductive additive are not shown). Briefly, during discharge the electrons move from the metallic collector (foil) across the conductive paths (mostly across conductive additives) to each individual active particle (step A in Figure 1a). Ions are provided from the electrolytes side. Both electrons and ions are simultaneously inserted into the active particles (step B in Figure 1a). Finally, ions and electrons move simultaneously into the inner of active particles (step C). However, this movement of ions and electrons inside active particles might proceed in very different ways, depending on the system. For example, in the conventional $\mathrm{LiCO}_{2}$ (LCO) cathode, this movement can be described as rather simple chemical diffusion process (at least in certain potential range of operation). At the other extreme, in $\mathrm{LiFePO}_{4}$ (LFP) electrode, this movement involves complicated phase transformations such as spinodal decomposition. Furthermore, these reactions may depend on particle size, reaction rate and other parameters. Due to these differences we will in continuation include typical results on both extreme insertion materials, LCO and LFP.

First we focus on a typical impedance spectrum of a LFP electrode (Figure 1b). We see two main features in this figure: a red high-frequency (HF) arc and a blue low-frequency (LF) line. Additionally, we may see that $\mathrm{HF}$ arc does not approach the origin of graph (the 0,0 point) but forms a small intersection at extrapolated highest frequencies. This part (small intersection) is usually ascribed to the resistance of electrolyte. As this resistance is comparatively small, it usually does not represent a big contribution to the total impedance of cell. The situation may change under high rates where the species from electrolyte may be consumed or formed at very high rates. This case, however, is not considered here. Thus it remains to find the origin of the other two features (HF arc, LF line). One way to find the meaning of these features and correlate them to the general scheme in Figure $1 \mathrm{a}$ is to look into previous results published by other authors and assume the present case is similar 
to previous ones. Another approach, which is more consuming but also much more reliable, is to make dedicated experiments that will reveal the actual correlation between the scheme in Figure $1 \mathrm{a}$ and the measurement in Figure $1 \mathrm{~b}$.
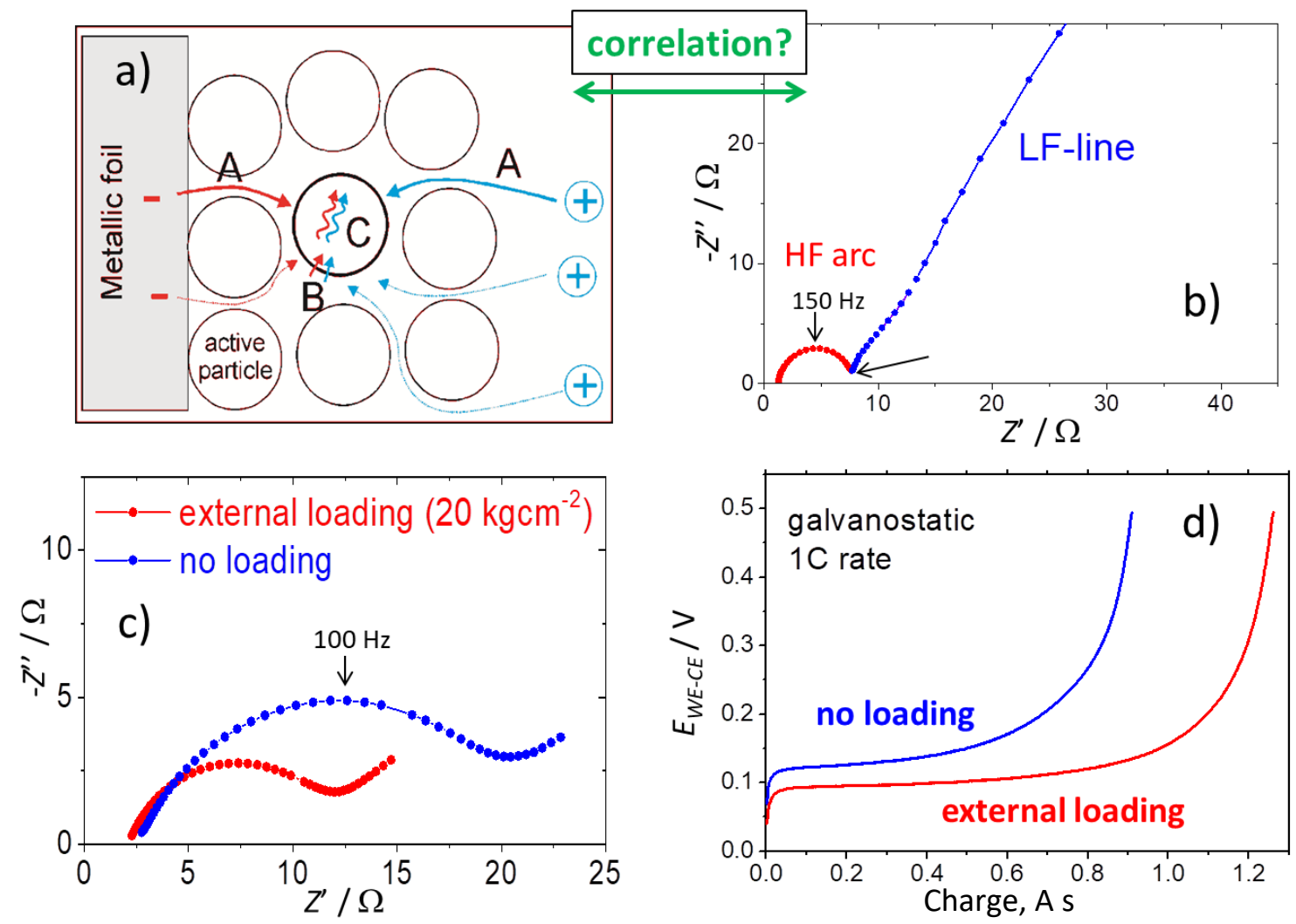

Figure 1. a) Three main steps of transport/reaction mechanism in insertion electrodes; b) typical impedance response of lithium iron phosphate (LFP) electrode; c) effect of external loading (mechanical pressure) on the HF impedance arc of LFP electrode in pouch cell (soft cell enabling application of external mechanical load); d) effect of external loading on cell capacity. The figure presents selected unused results from the study published in ref. [3].

An example of such dedicated experiment is shown in Figure 1c. The pouch cell containing two LFP cathodes was pressed externally and the impedance response measured before and after exerting the pressure (for details see ref. [3]). As regard the impedance measurements, they were carried out with different equipments, depending on the type of cell and frequency range investigated: in the frequency range of $1 \mathrm{MHz}$ to $20 \mathrm{~Hz}$, we used Hewlet Packard 4284A instrument, while in the frequency range of $100 \mathrm{kHz}$ to $0.001 \mathrm{~Hz}$ we used Solartron SI 1260 coupled with PAR EG\&G 283 potentiostat/galvanostat. The galvanostatic characterization was performed using VPM3 Potentistat/Galvanostat at room temperature. We may see that the use of mechanical pressure on soft pouch cell decreases considerably the size of HF arc. Making many sets of such experiments and varying the pressure in a controlled and repeatable way [3], we assumed that HF arc might correspond to some contacting, as a contact resistance is known to be dependent on the pressure between contacting phases. However, looking at Figure 1a, one sees two types of contacts in a typical battery cell: (i) the one between the current collector (here aluminium foil) and the electrode composite, and (ii) the contacts between the individual active particles constituting the electrode composite. Thus a further dedicated experiment was required, as exemplified in Figure 2.

From Figure 2 we can see that if we apply a conductive paste between the current collector and the composite electrode, the HF arc more or less disappears - even if no pressure is exerted on the 
pouch cell [3]. This experiment indicates quite convincingly that the HF arc must be due the poor contact between the current collector and the electrode composite. After application of $\mathrm{Ag}$ the contact becomes more or less invisible due to improved electronic conductivity across that contact. This assumption was additionally confirmed by several other sets of experiments as discussed in our previous paper [3].

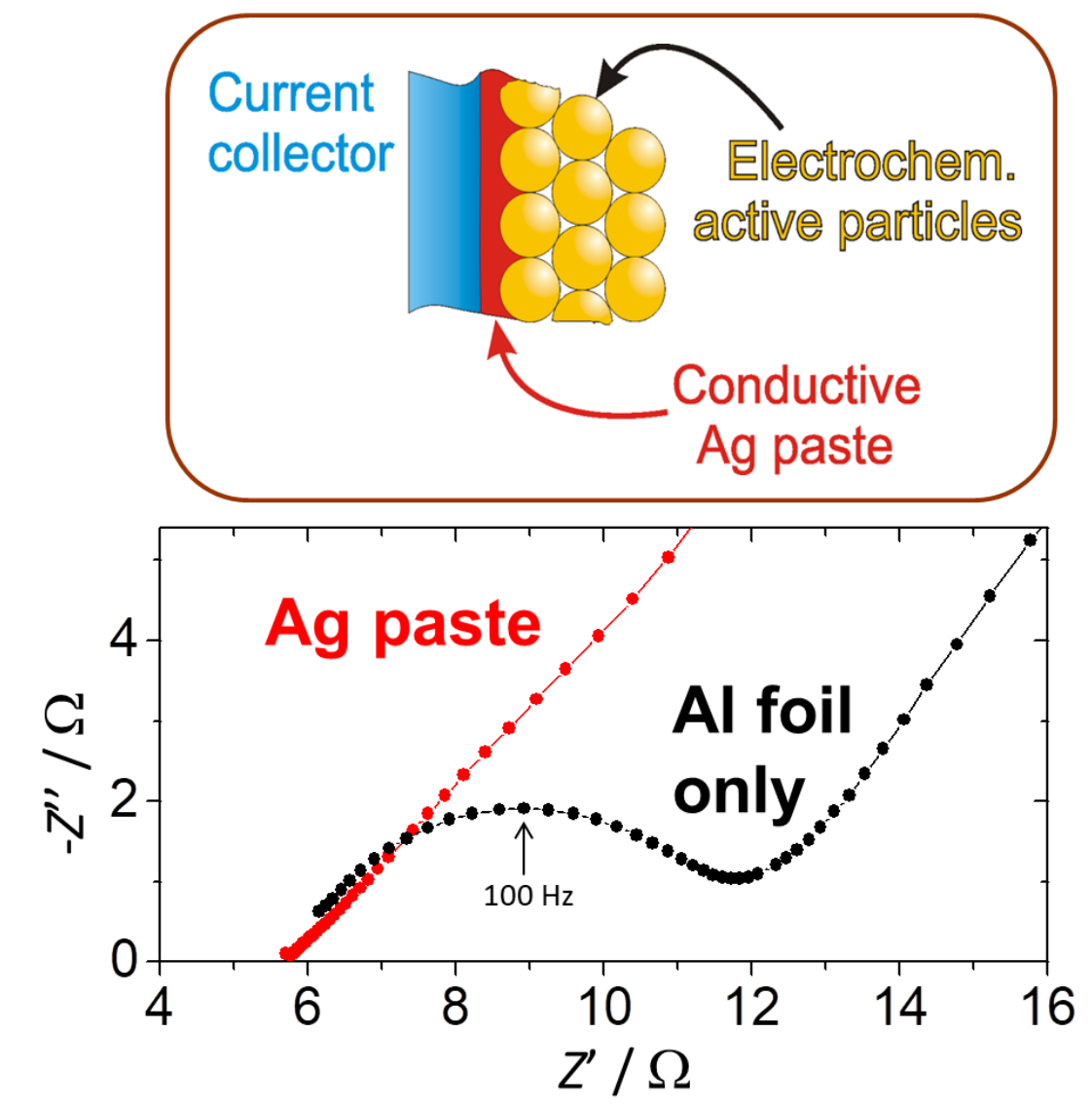

Figure 2. Improving (decreasing) the contact resistance between aluminium substrate and electrode composite by applying conductive Ag paste to that interface

(adapted and redrawn based on the results of ref. [3]).

Based on the results shown and discussed above, one might wonder if the finding about the origin of $\mathrm{HF}$ arc also holds for other cathode systems, for example the more conventional lithium cobalt oxide, LCO. Thus we performed a similar experiment using the variable external pressure also on the LCO system (Figure 3a) (for details see ref. [4]). However, already a quick look at the typical spectrum, we notice that LCO electrode gives an impedance response that contains two arcs, denoted as A and B. At least two obvious questions arise: which of the arcs is due to the contact between the collector and electrode composite (if any) and what is the meaning of the other arc? The experiment with the variable pressure reveals that only the size of arc A significantly changes with pressure whereas the size of $\operatorname{arc} B$ is more or less pressure-independent. This strongly indicates that the arc A must be due to the contact resistance - similarly as HF arc in the case of LFP. Indeed this assumption was confirmed by a more detailed analysis, as shown in our previous work [4]. Still, the question remains - what is the meaning of arc B? Based on the peak frequency and the associated capacitance we assumed that one explanation could be that the arc B represents the resistance of insertion reaction (step $B$ in Figure 1a) coupled with the double layer capacitance of the interfacial region between the electrolyte and active particles. However, this assumption needed to be confirmed/refuted by some dedicated experiments. 


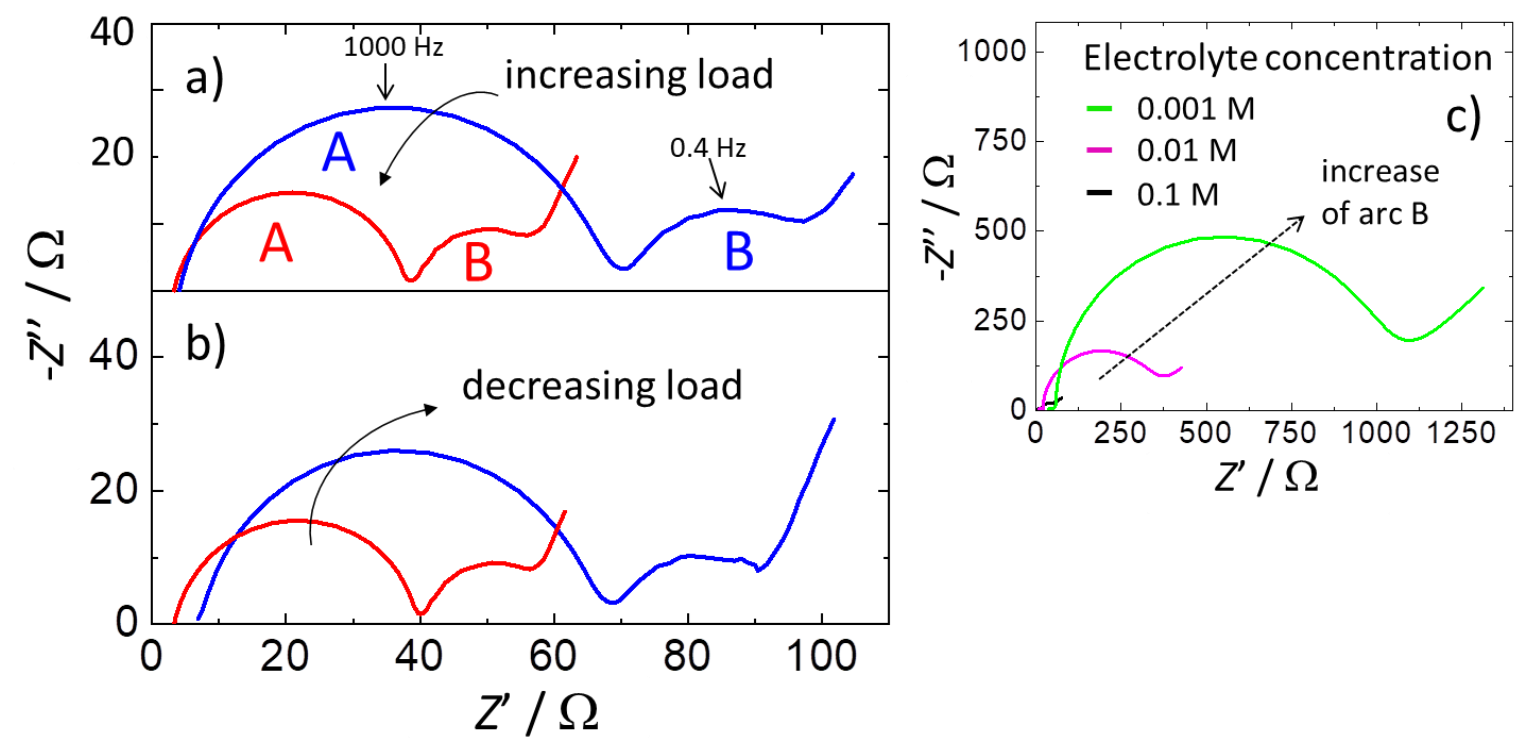

Figure 3. $\boldsymbol{a}, \boldsymbol{b})$ Typical effect of external loading (mechanical pressure) on the impedance response of a lithium cobalt oxide (LCO) electrode. Note the absence of effect on arc "B«. c) Effect of changing the electrolyte concentration on arc B of $L C O$ electrode. The electrolyte consisted of a LiPF 6 salt in EC:DEC (ethylene carbonate/diethyl carbonate) (1:1 by volume).

An example of such experiment is shown in Figure 3c. We prepared several cells that contained as similar as possible LCO electrodes but in each cell the concentration of electrolyte was different. We found that any decrease in the electrolyte concentration led to a significant (and systematic) increase of $\operatorname{arc} B$. Simultaneously, the electrolyte concentration had a significant impact on the peak frequency of arc $B$ and thus on the associated capacitance. A more detailed analysis showed that all the trends were consistent with the main assumption, namely that arc $B$ was due to insertion reaction coupled with double layer capacitance [4].

Based on some additional dedicated experiments and analyses shown in previous works [5-7], we could construct a transmission line (Figure 4) that could describe quite accurately all of the measured impedance spectra of insertion electrodes.

The physical meaning of individual transmission line elements is described in Figure 4. Based on this description we can say that in both materials, LFP and LCO, the HF arc is due to the coupling between elements $r_{0}$ and $c_{0}$, i.e. the resistance and capacitance due to the contact between current collector and electrode composite. Furthermore, one can conclude that arc B in the case of LCO is due to the sum of discretized elements $r_{2, i}$ and $c_{1, i}$, i.e. the resistance due to insertion reaction coupled with the capacitance due to the double layer between the electrolyte and the active particles. As mentioned before, resistance due electrolyte, $r_{4, i}$, appears as the high-frequency intersection.

What has not been resolved, however, is the meaning of the low-frequency line observed especially clearly in the case of LFP (Figure 1b, blue line). If we look closer to that part of impedance response, we in fact see a sort of hump at the beginning of line. Also, we can see that the line is quite inclined, i.e. it is not vertical. If it was vertical and without a hump we could assume that it must be due to some big capacity in system, such as the chemical capacity of insertion material itself. If the inclination was close to 45 degrees, we could ascribe it more or less directly to a Warburg impedance, possibly due to the chemical diffusion in the solid active particles, as indeed indicated in the proposed transmission line, i.e. the element $Z_{w, i}$ in Figure 4. 

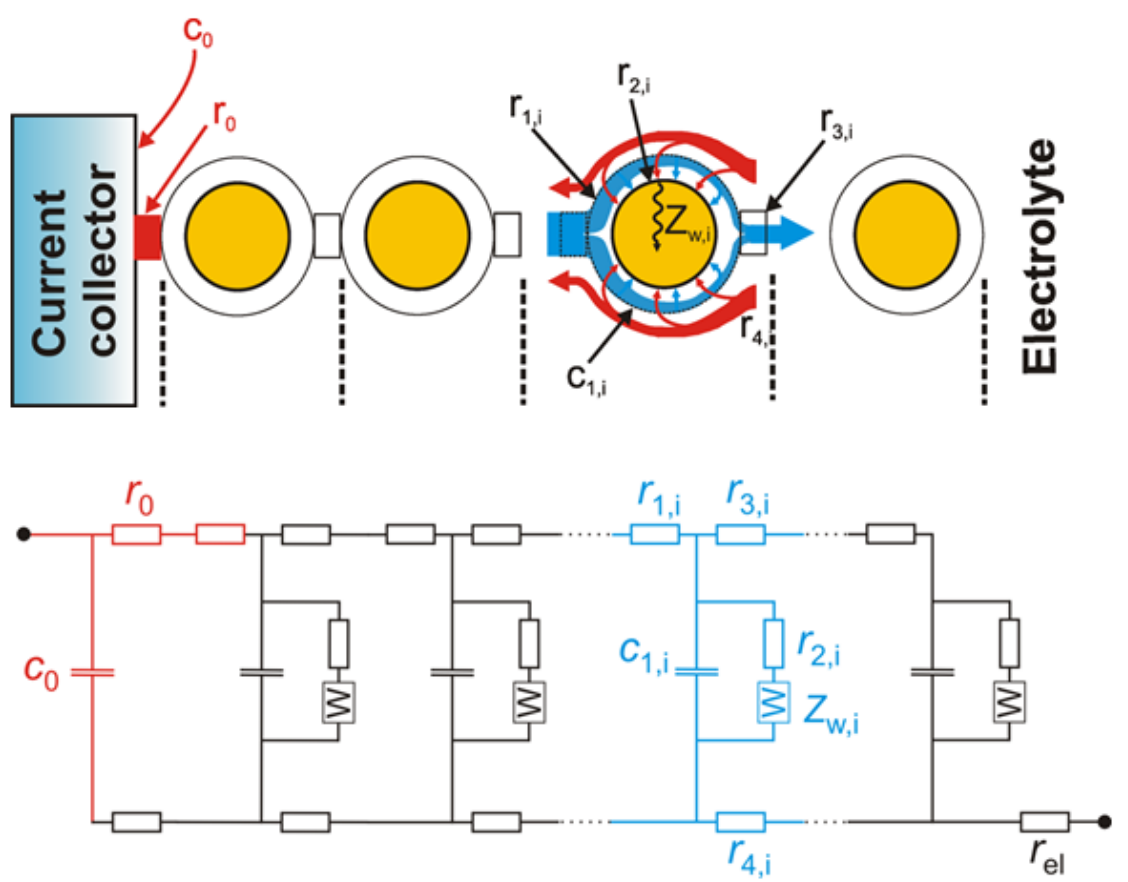

Figure 4. Top: a scheme of a typical porous insertion electrode indicating resistive and capacitive contributions to impedance response: $\mathrm{c}_{0}$ - capacitance due to contact between current collector and electrode composite; $r_{0}$ - resistance due to contact between current collector and electrode composite; $\mathrm{r}_{1, i}$ - electronic resistance of carbon coating; $\mathrm{c}_{1, i}$-capacitance due to electrolyte/active particle interface;

$r_{2, i}$ - resistance due to insertion reaction; $r_{3, i}$-resistance due to contact between active particles;

$r_{4, i}$-resistance due electrolyte; $Z_{w, i}$ - Warburg impedance due to diffusion of active species inside active particles; $\mathbf{r}_{\boldsymbol{e}}$-electrolyte resistance.

Bottom: a transmission line model corresponding to the proposed scheme.

Again, to get further insight into the meaning of this LF line we performed additional systematic experiments. For example, Figure 5 a shows that the inclination of the LF line changes with state of charge of the cathode whereby it approaches the expected 45-degree case as the SoC increases towards the medium values (half of total charge). An analysis based on the proposed transmission line showed that such a behavior could mean that during charging the averaged diffusion coefficient inside active particles is changed [4].
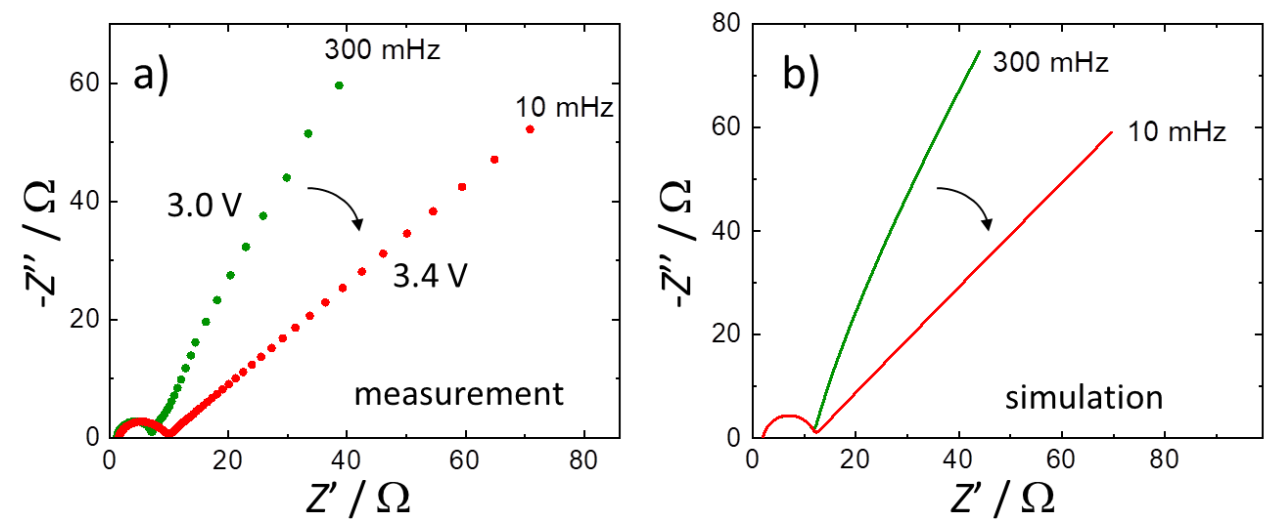

Figure 5. Measured (a) and simulated (b) impedance spectra of LFP electrode at two different states of charge (corresponding to two different potentials). Note the decreasing angle as the SoC increases. The effect is reproducible and reversible which indicates a unique correlation between the angle and SoC.

The figure presents selected unused results from the study published in ref. [4]

Indeed such variation of diffusion coefficient was earlier shown by other authors for different insertion systems [5-7] and is generally attributed to the formation of non-ideal mixtures (occupied 
vs non-occupied sites with $\mathrm{Li}$ during insertion or de-insertion of lithium). However, a more mechanistic explanation that would correlate this variation with $\mathrm{SoC}$ is still missing so further model experiments are needed to fully understand this phenomenon.

As a whole, the information collected so far enables one to make several conclusions as regards the meaning of measured impedance features in insertion electrodes. Figure 6 summarizes such conclusions by connecting the regions in an insertion electrode where a given process occurs with the corresponding impedance features. The latter are, in turn, also uniquely connected to the elements of transmission line model. Such a scheme might represent a good starting point for studying any insertion material. However, similarly as shown above, at least some model experiments (systematic/dedicated experiments) need to be performed to confirm the validity of the scheme and, possibly, identify specific feature(s) for given system.
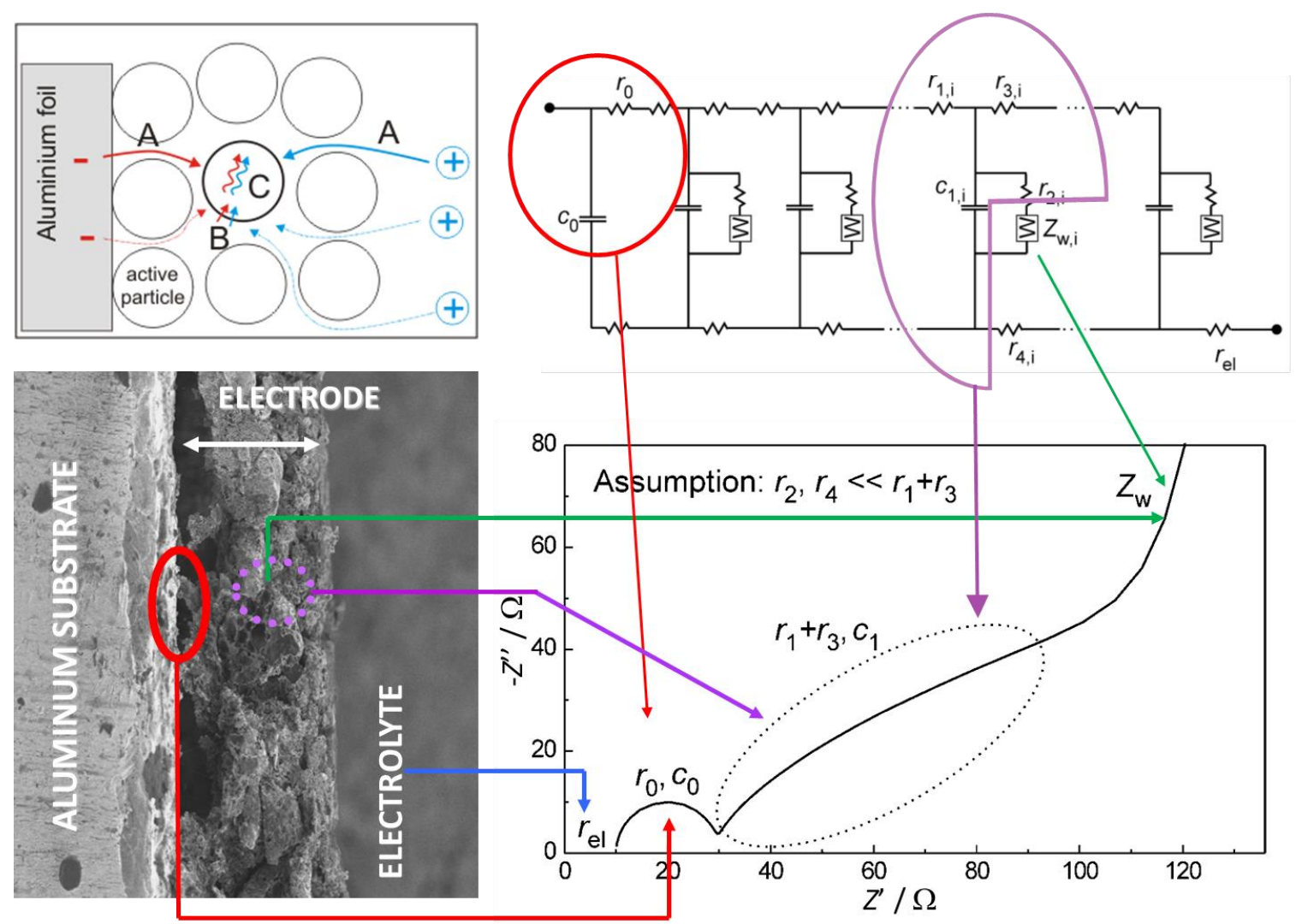

Figure 6. Left: Schematic and SEM image of a typical insertion electrode; Right: transmission line model and general simulated impedance response of insertion electrode. The correlations between the image, transmission line and impedance response are clearly indicated.

\section{Litium sulfur system: sulfur electrode (porous carbon in contact with polysulfides)}

Li-S battery is a highly promising candidate to replace the current lithium ion systems [8-10]. However, there are still several open questions regarding the very basic operation of this complex system. Impedance spectroscopy could help answer some of them. As mentioned before, significant progress can only be expected if many sets of dedicated experiments are carried out and the results are combined with the findings obtained using other methods. Here we show how it is possible to get additional insight into selected cathode mechanisms by using different electrode morphologies such as a flat electrode or a porous electrode with well-defined morphology.

The cathode of Li-S system is usually composed of sulfur that is embedded into the pores of a highly porous conductive substrate such as a porous carbon. During cell operation the initial solid sulfur gradually electrochemically reduces into soluble polysulfides with progressively shorter 
chains. Thus, most of the capacity obtained represents the electrochemical reduction of polysulfide species. As many of these species are soluble, they can move away from the carbon surface. If so, they can later be reduced only if they diffuse back to the surface. This complicates the chargedischarge mechanism which is also reflected in the corresponding impedance spectra. Figure 7 shows a typical development of impedance spectra during discharge of a conventional Li-S cell. The development during charging (not shown) is similar, only going to backward direction, as expected for a reversible reaction. In any case, what we can see in Figure $7 \mathrm{~b}$ is that most of the measured impedance responses are dominated by the low frequency response consisting of tail (a curved line) with progressively higher impedances. An analysis of this response appears to be rather difficult and ambiguous. The development during charging (not shown) is similar, only going to backward direction, as expected for a reversible reaction. In any case, what we can see in Figure $7 \mathrm{~b}$ is that most of the measured impedance responses are dominated by the low frequency response consisting of tail (a curved line) with progressively higher impedances. An analysis of this response appears to be rather difficult and ambiguous.
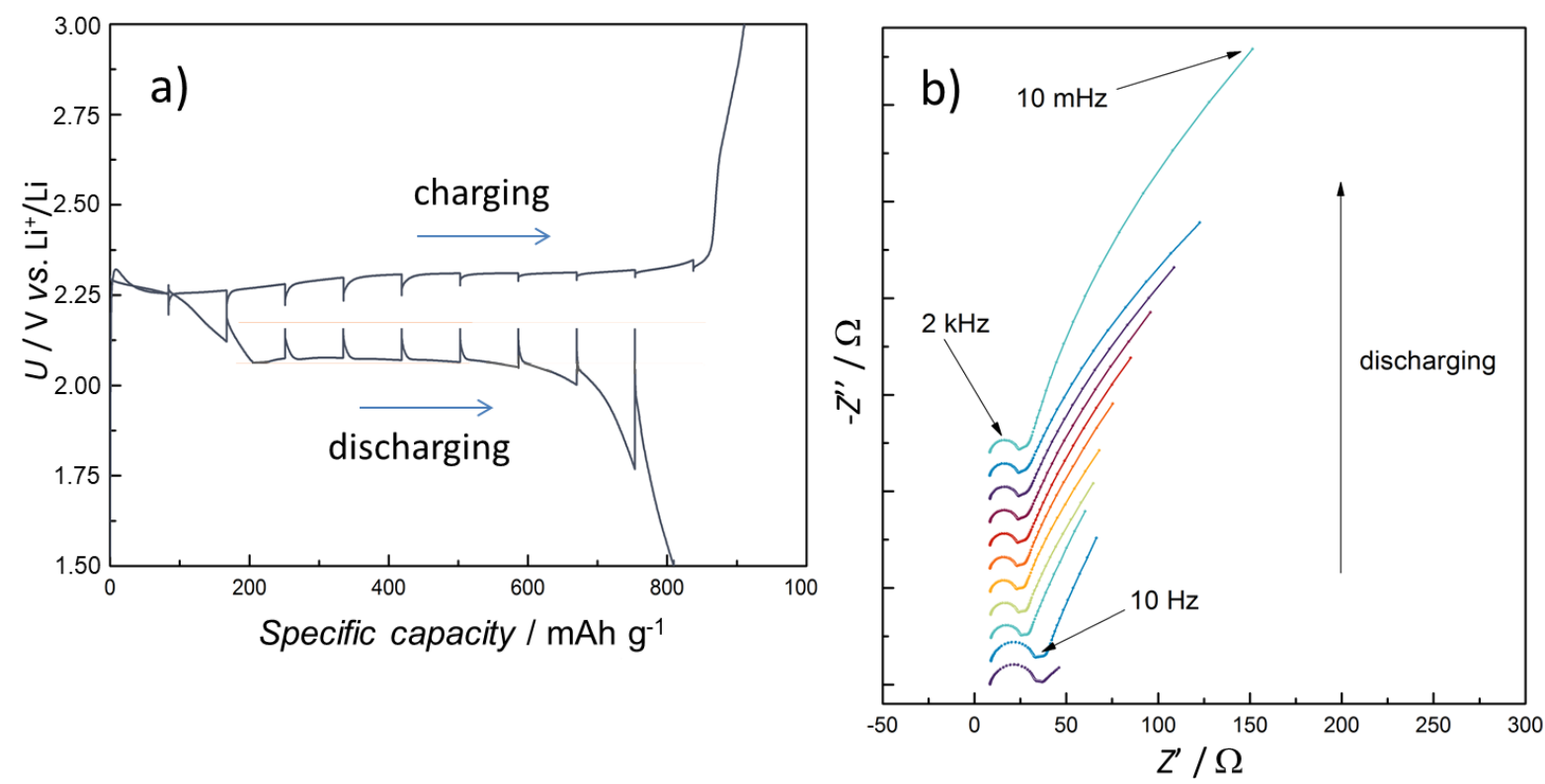

Figure 7. a) Typical charge-discharge curve of Li-S cell. The spikes represent the points where the cell operation was stopped and impedance spectra measured (after stabilization). The corresponding spectra obtained during the discharge are shown in panel b). The ENSACO $350 \mathrm{G}$ carbon (Imerys) and a corresponding sulfur composite (ENSACO 350G/S) were prepared in a C:S=1:2 mass ratio. The composite was mixed with a Printex (Degussa) conductive additive and a PVdF binder (8:1:1) in (NMP N-methyl-2-pyrrodione). Sulfur loading was approx. $1 \mathrm{mg} / \mathrm{cm}^{2}$. The electrolyte was $1 \mathrm{M}$ LiTFSI (lithium bis(trifluoromethanesulfonyl)imide) in TEGDME (triethylene glycol methyl ether):DOL(dioxolane) 1:1 (v:v). The separator was Celgard 2400. The figure presents selected unused results from the study published in ref. [11]

We have thus proposed to simplify the conventional Li-S cell in three ways [11,12]: (i) we replaced the porous cathode with a flat glassy carbon, (ii) we removed the lithium anode and used another glassy carbon to form a symmetrical carbon-carbon cell, (iii) instead of using sulfur we used different polysulfides to study the various stages of charge-discharge process. Typical results and a basic analysis are shown in Figure 8.

A comparison between the impedance spectrum shown in Figure $8 \mathrm{c}$ and the spectra displayed in Figure $7 \mathrm{~b}$ shows several pronounced differences. Firstly, the impedance spectrum in Figure $8 \mathrm{c}$ consists of a much bigger high-frequency arc (several thousands $\Omega$ ) as opposed to the much smaller (50-100 $\Omega$ ) arc in conventional cell. 

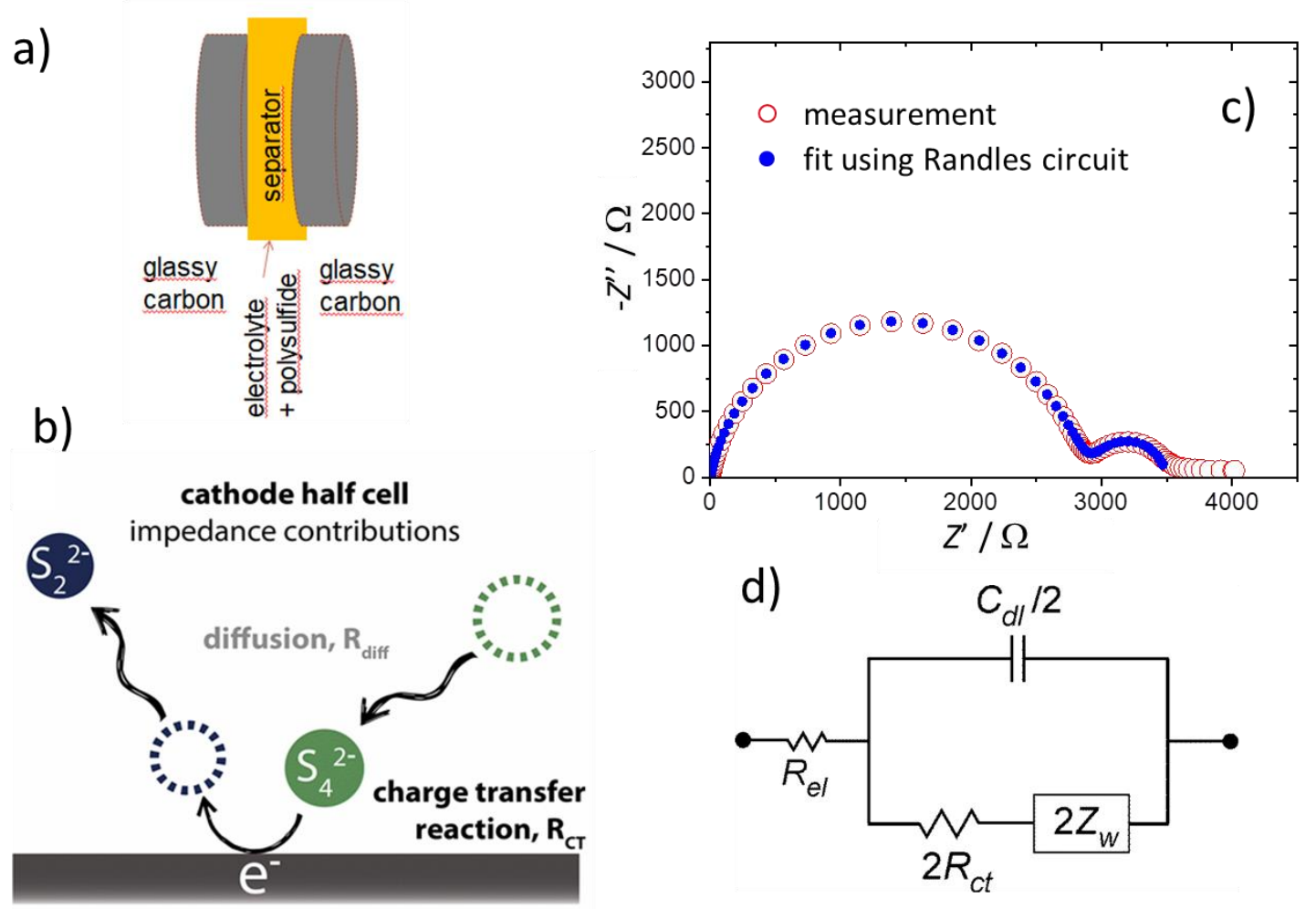

Figure 8. a) Schematic presentation of the flat symmetrical cell used to study the basic reaction-diffusion properties of various polysulfides; b) A typical reaction-diffusion scheme that could be studied in proposed cell (e.g. reduction of $\mathrm{S}_{4}{ }^{2-}$ to $\mathrm{S}_{2}{ }^{2-}$ ); c) measured impedance spectrum (red open circles) of reaction under (b) and the corresponding fit (blue circles) using the Randles circuit shown in panel (d). Impedance spectra of the symmetrical glassy carbon cells were measured at OCV with the same voltage amplitude (10 $\mathrm{mV}, \mathrm{rms})$. The low-frequency limit was varied and extended down to $0.1 \mathrm{mHz}$. Adapted partial results from ref. [11]

Secondly, at low frequencies the spectrum in Figure 8c consists of rather small arc that extends to poorly defined shape at lowest frequencies. Analysis [11] showed that the latter feature was an artefact - most likely due to tendency of polysulfides to disproportionate. By contrast, the other features (big and small arc) could very well be fitted with the conventional Randles circuit as demonstrated by the good match between blue and red circles. Making many such experiments involving different polysulfides, we could determine quite accurately the main reaction (exchange current density, $i_{0}$ ) and transport (chemical diffusion coefficient, $D_{\text {chem }}$ ) parameters, as shown in detail in our previous paper $[11,12]$.

Once the basic intrinsic reaction and transport parameters of different polysulfides were known, we could move back to more complex electrode configuration. Specifically, we retained the symmetrical cell configuration but replaced the flat electrodes with porous ones. We used porosity of different degrees in order to decouple the effect of porosity itself from the other electrode parameters. In this brief overview we only show selected results on unconventional porous electrodes consisting of a carbon felt with intermediate level of specific surface area $\left(15 \mathrm{~m}^{2} \mathrm{~g}^{-1}\right)$ (Figure 9). Room temperature impedance spectra of the cells were measured on VMP3 potentiostat/galvanostat (Bio-Logic) at OCV in the range from $1 \mathrm{MHz}$ to $0.1 \mathrm{mHz}$ (or $20 \mu \mathrm{Hz}$ ) with 10 (5) points per decade and an amplitude of $10 \mathrm{mV}(\mathrm{rms})$.

The general impedance response of porous electrodes involving surface reaction and diffusion in pores (corresponding well to the situation in the present carbon-polysulfide system) has been derived analytically $[13,14]$. Two typical impedance spectra predicted using the analytical expression are displayed in Figure 9b. 
a)
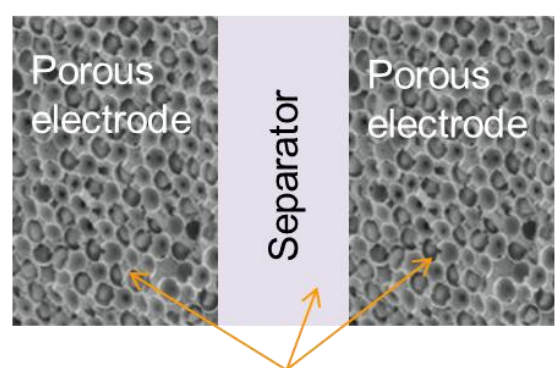

Polysulfides, $\mathrm{Li}^{+}, \mathrm{A}^{-}$

solvent

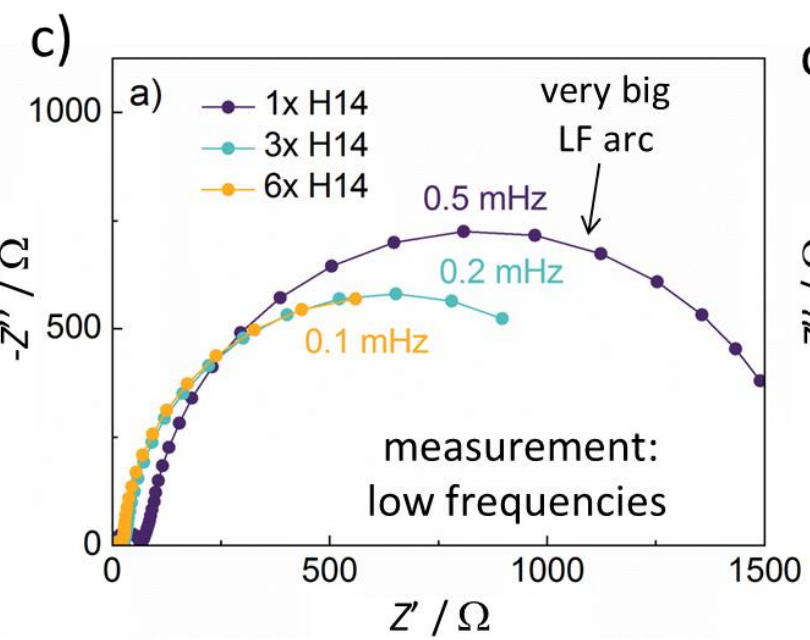

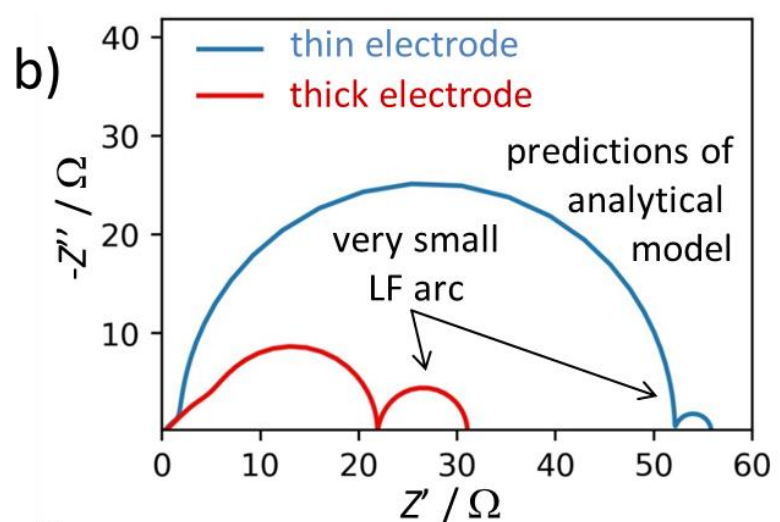

d)

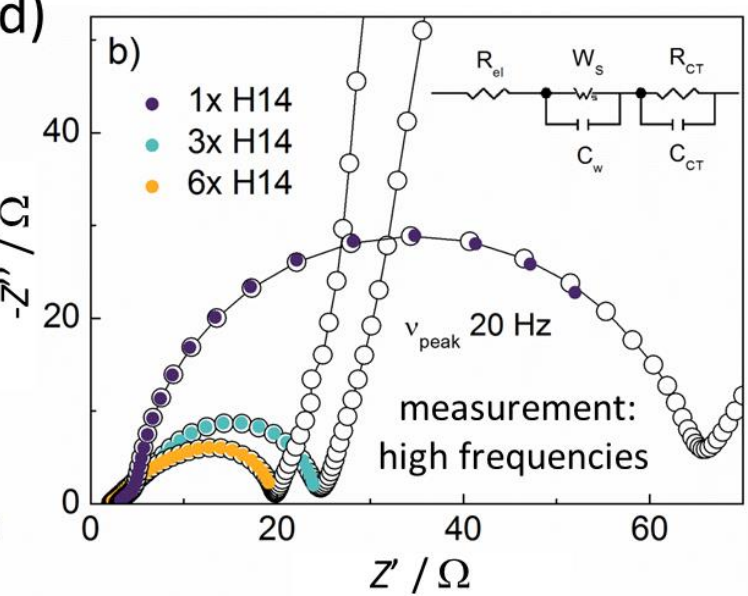

Figure 9. a) Schematic of a carbon-carbon cell where porous carbon felt $\left(15 \mathrm{~m}^{2} \mathrm{~g}^{-1}\right)$ was used as a model porous electrode; $\boldsymbol{b})$ Theoretical impedance response of a porous battery electrode (with surface reaction) as predicted by the analytical model in ref. [14]; c) measured impedance response of symmetrical carboncarbon cell with polysulfides as active substance - low frequencies; d) measured impedance response of symmetrical carbon-carbon cell with polysulfides as active substance - high frequencies (adapted from ref.

[15]); attempts to fit the spectra using ordinary Voigt circuit (colored parts) were not entirely successful

It can be seen that impedance spectra consist of a high frequency arc with more or less expressed 45-degree feature at the highest frequencies. At low frequencies there is an additional arc but typically with a smaller size. Also, we can see that the size of both arcs depends on the electrode thickness: the size of HF arc decreases with thickness, while the size LF arc increases. Comparing the theoretical predictions to the actual measurements we can see that $\mathrm{HF}$ arc follows quite well the predicted trends. However, there is a large mismatch between the prediction and the measured behavior of LF arc: firstly, it is much bigger (about 2 orders of magnitude) than the predicted arc and secondly, its size does not increase with electrode thickness. Further analysis in which we systematically changed the concentration of catholyte solution, the type and thickness of separator etc. [15] revealed that in fact, the theoretically predicted low-frequency arc was masked by the response of catholyte-soaked separator. In other words, the diffusional processes occurring in the separator prevailed over those occurring in the porous cathode itself. As we could not find any model for this particular situation, we built-up our own model (Figure 10) based on the well-known transmission line approach developed for porous electrochemical systems [16].

The validity of model was first checked for the case where the response of separator was neglected (set to zero). We found a complete correspondence between the model shown in Figure 10 and the recent analytical solution [14]. Then the response of separator was plugged in, and the predictions of the whole model compared to the actual measurements. Typical simulations are displayed in Figure 11. 


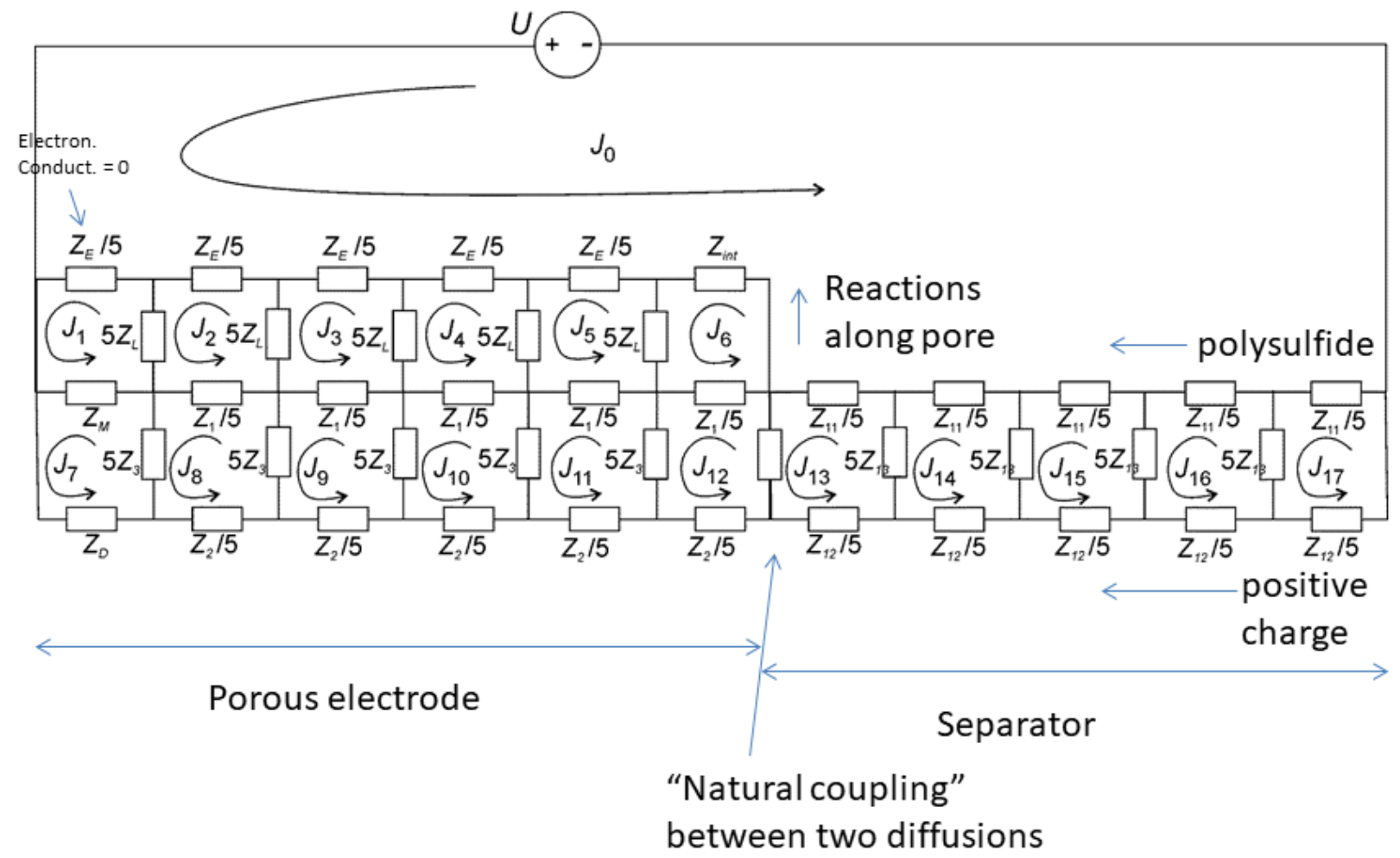

Figure 10. General transmission line scheme that takes into account diffusion in separator (right part, as denoted), diffusion in porous cathode (elements $Z_{1}, Z_{2}, Z_{3}$ ) and reaction in porous cathode (elements $Z_{L}$ ). Electronic conductivity of carbon matrix (elements $Z_{E}$ ) is set to zero for simplicity (however, in principle could be of any value). More detailed meaning of all the elements can be found in our previous article [15].

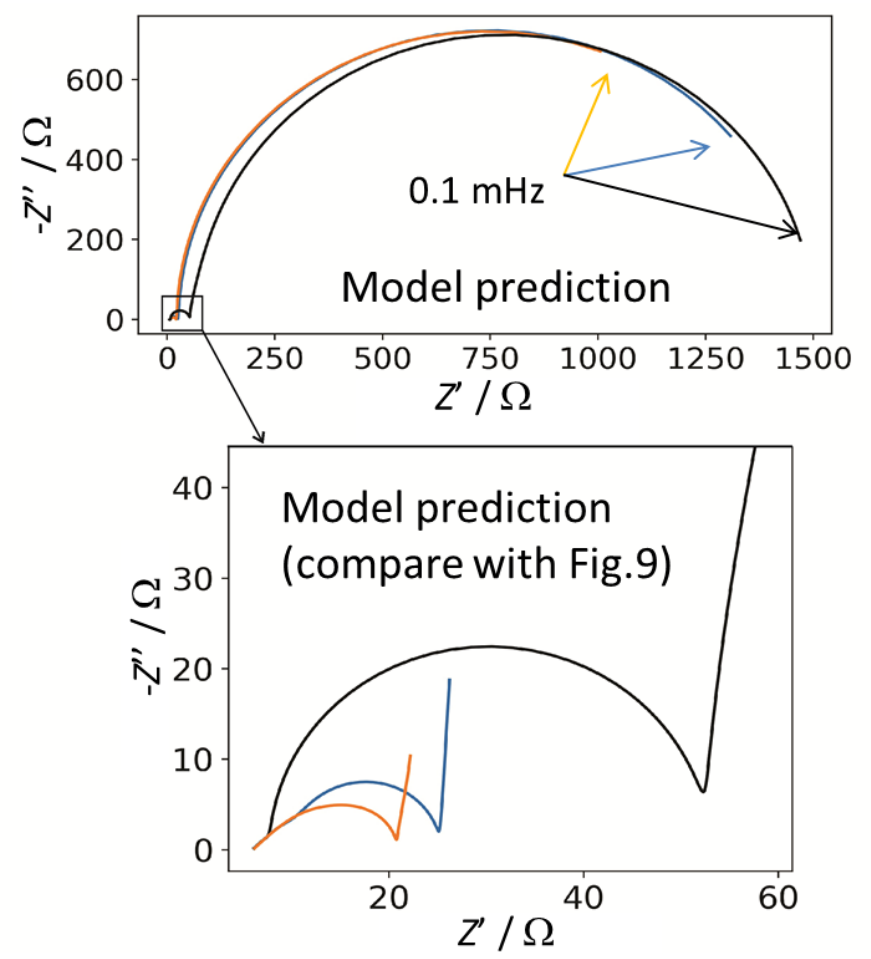

Figure 11. Low (top) and high (bottom) frequency response of porous cathode in contact with separator as predicted by the model in Figure 10.

We can see that now the low-frequency response is very similar to the measurements (cf. Figure $9 \mathrm{c}, \mathrm{d}$ ) while, as expected, the good prediction of HF arc from the analytical solution is also retained. In fact, a more detailed analysis shows that the chemical capacity of LF arc (related to its peak frequency) is a sum of the values for the porous cathode and porous separator. This theoretical prediction was fully confirmed in all measurements on different cells carried out in this study. 


\section{Metallic lithium anode}

Unlike the sulfur cathode of the Li-S system, the metallic lithium anode has been subject of intense investigation for more than 40 years $[17,18]$. In organic electrolytes, lithium gets spontaneously passivated by a surface layer that conducts Li ions but is more or less non-conductive for electrons and hence its widely accepted name "Solid Electrolyte Interface» (SEI) [17-20]. However, the nature of SEI as well as the surface of Li anode in general is significantly modified during repetitive cycling $[21,22]$. Various proposed mechanisms exist that describe Li morphology obtained after certain number of cycling. Here we assume and treat only the most general features that are common to many investigations: (i) continuous dissolution and re-deposition of lithium leads to a porous morphology of superficial lithium (called dendrites) as the dissolution-deposition process does not proceed uniformly across the surface; (ii) the porous Li structures (dendrites) are passivated on their surface with SEI-like film; (iii) there are at least two types of dendrites, the socalled »live dendrites" and "dead dendrites«; the former still contain a significant amount of metallic lithium, whereas the latter contain a small amount of electrically disconnected lithium or even no lithium but only SEI-like products.

Due to the complex surface morphology of cycled lithium anode, it is not surprising that impedance spectra are also quite complex such as demonstrated in Figure 12. In that experiment, a symmetrical $\mathrm{Li}$-Li cell was cycled as indicated on the left part of the figure. The cells for this experiment were made according to the pouch technology using two lithium electrodes and Celgard 2320 separator.
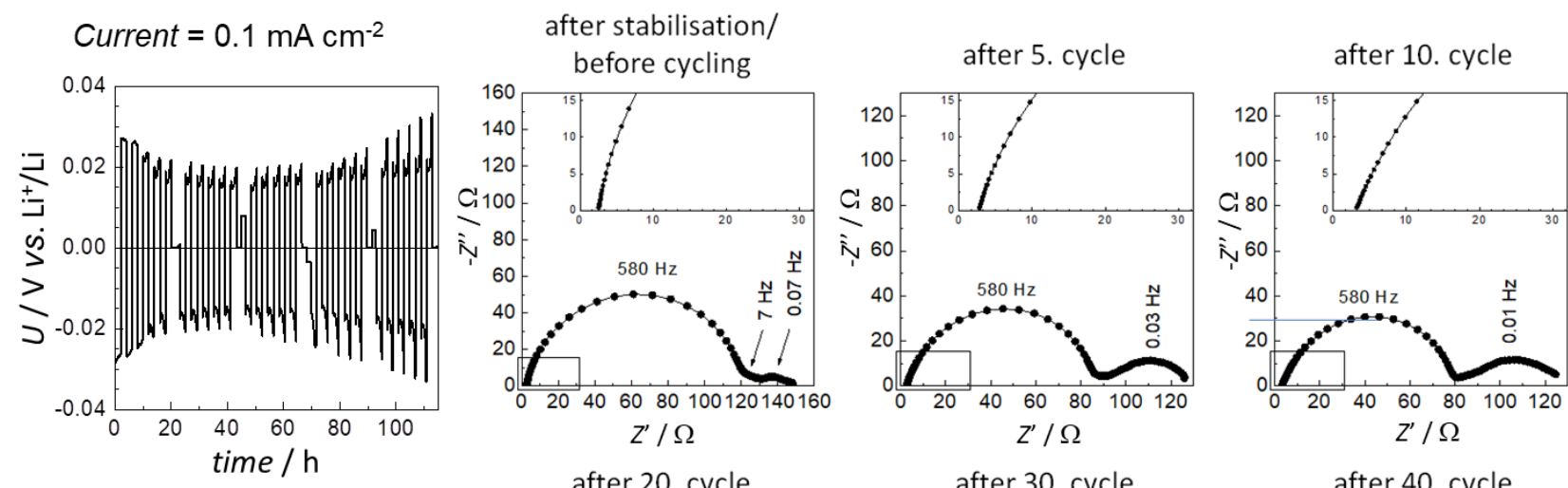

$20 \mu \mathrm{L}$ of $1 \mathrm{M}$ LiTFSI in TEGDME:DOL

Celgard 2320
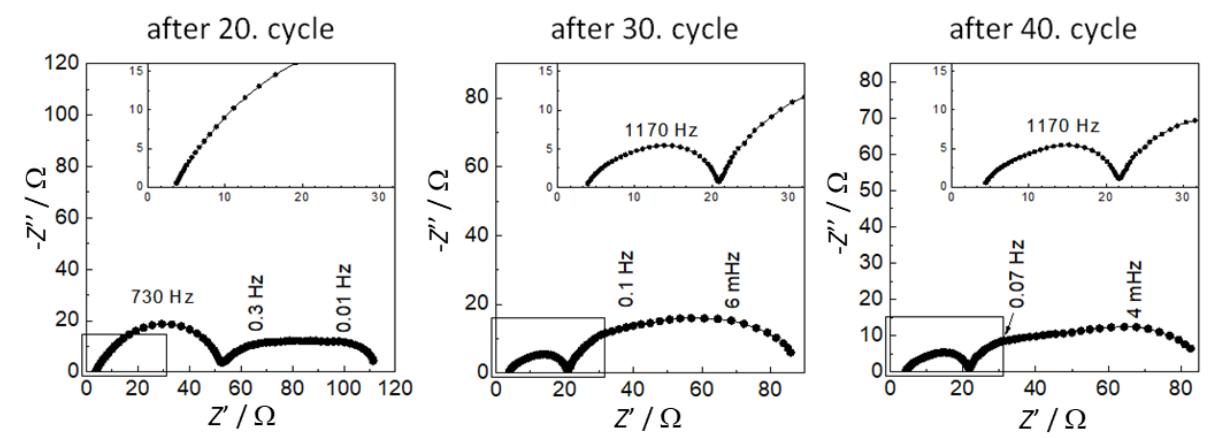

Figure 12. Development of impedance spectra with the number of cycles (shown on top left) on symmetrical Li-Li cell. For clarity only selected cycle numbers are displayed. See development of unusual shapes at low frequencies representing the development of dendrites. The figure presents selected unused and/or adapted results from the study published in ref. [23]

The electrolyte used was $20 \mu \mathrm{L}$ of $1 \mathrm{M}$ LiTFSI in TEGDME:DOL 1:1 (v:v). The electrochemical experiments on the cells were done with a combination of impedance spectroscopy measurements and stripping and deposition cycles on Li| | Li symmetrical cells. The cells were connected to a VMP3 Biologic potentiostat/galvanostat within 10 minutes of cell assembly. They were left to stabilize for 24 hours, during which impedance spectra were measured. After this initial period, Li||Li cell 
underwent stripping and deposition at a constant current rate for 2 hours. The current for the discharge/charge cycles was either $0.2 \mathrm{~mA}$ ("low current cells") or $1.0 \mathrm{~mA}$ ("high current cells"). After stripping and deposition of lithium, the cells were left to relax for 15 minutes, after which impedance spectra were measured. All of impedance spectroscopy measurements were done in the range of $1 \mathrm{MHz}$ to $1 \mathrm{mHz}$ with an amplitude voltage of $10 \mathrm{mV}$ (rms).

As seen from Figure 12, during cycling the impedance response of the cell changes significantly. HF arc is gradually decreasing whereas LF arc transforms into an extended shape typical for cases where several processes closely overlap. In order to check the validity of such measurements (that potentially include some uncompensated dynamics and additionally is carried out to very low frequencies), we carried out a couple of standard testings such as (i) measurements at different values of excitation signal (testing the linearity of response), (ii) repetition of measurements at given steady state conditions (checking the time invariance of the system) and (iii) Kramers-Kronig analysis (for details see ref. [23]).

Based on the assumptions $\mathrm{i}$-iii stated above we constructed a transmission line that captures the main known morphological features of Li-Li cell (Figure 13).
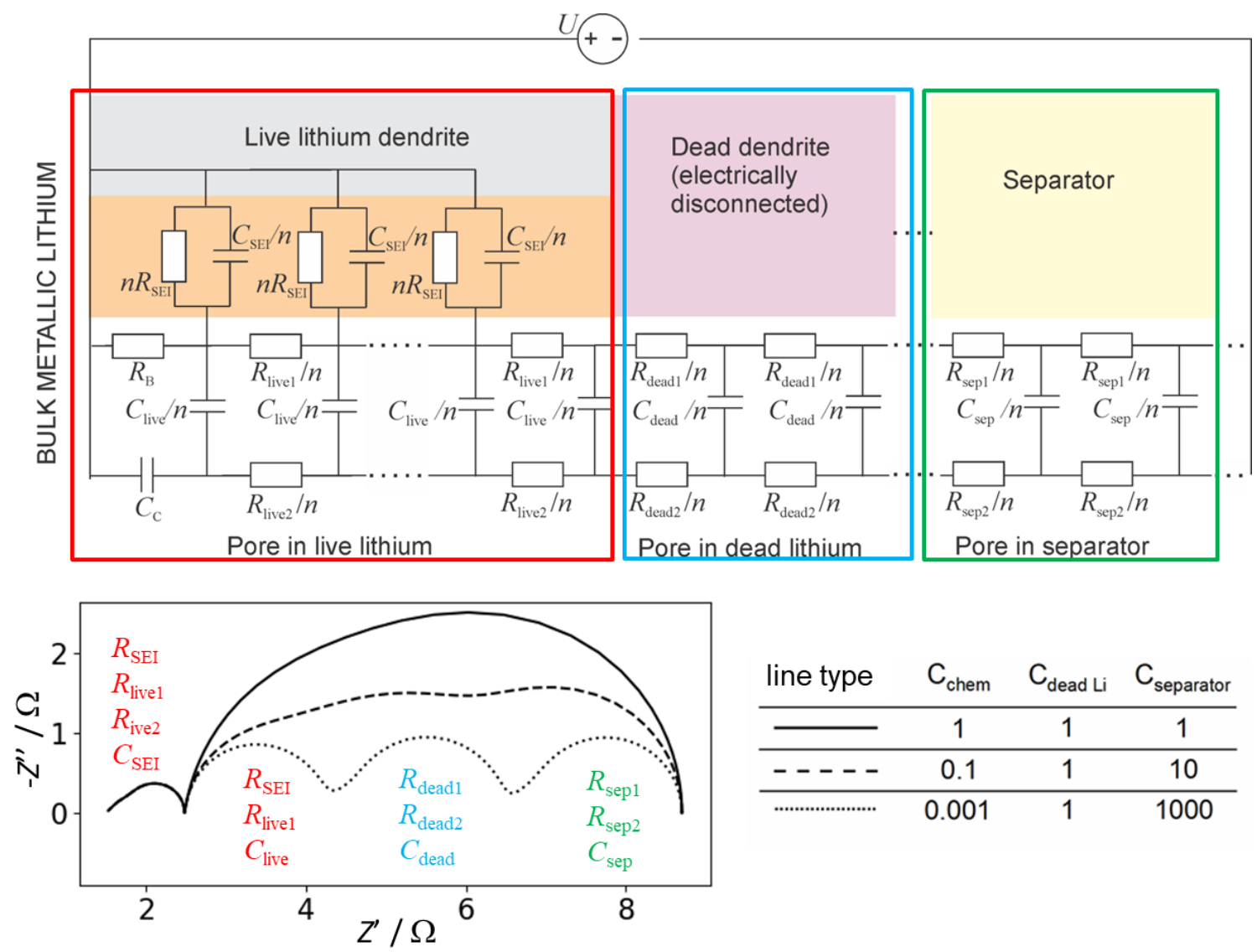

\begin{tabular}{cccc} 
line type & $\mathrm{C}_{\text {chem }}$ & $\mathrm{C}_{\text {dead Li }}$ & $\mathrm{C}_{\text {separator }}$ \\
\hline- & 1 & 1 & 1 \\
\hline----- & 0.1 & 1 & 10 \\
\hline$\cdots \cdots \cdots \cdots . . . \cdots$ & 0.001 & 1 & 1000
\end{tabular}

Figure 13. Top: Transmission line model proposed for lithium anode containing dendrites. Live and dead dendrites are considered separately as they have different basic properties (see main text). Bottom: Simulations using the proposed transmission line; different level of coupling between live dendrites, dead dendrites and separator are displayed; most measurements show a close coupling corresponding either to the dashed or solid graphs (compare with the shapes seen in Figure 12).

Briefly, the main feature that generates the first and the second dotted arc are the porous live dendrites. It is quite remarkable that in fact a porous live lithium filled with electrolyte is conceptually almost identical to a porous carbon electrode containing polysulfides. This is due to the fact that in both cases the reaction proceeds on a conductive porous surface whereas at low 
frequencies, diffusion of active species inside the pores (the second arc) is the predominating process in both cases. However, the main difference is that the cycled lithium electrode also contains some dead dendrites where no surface reaction can take place so only diffusion across the pores is seen (see second compartment of scheme in Figure 13). This part contributes to another, third arc which however is quite strongly coupled to the second one (because in both cases the main diffusional processes are similar). Finally, at lowest frequencies (the third compartment in Figure 13) the arc due to diffusion in separator occurs. As the thickness and porosity of the separator are similar as in live or dead lithium, this arc is also strongly coupled to both dendritic features. As a whole, this strong coupling gives an extended shape as indeed observed in the actual measurements (compare the simulations in Figure 13 with some of the measured shapes in Figure 12, especially the ones after $20^{\text {th }}, 30^{\text {th }}$ and $40^{\text {th }}$ cycle where dendrites have already fully evolved).

\section{Conclusions}

In this brief review we showed how impedance spectroscopy can be used for systematic investigation of selected mechanisms occurring in modern batteries. In the first part we focused on explanation of the main impedance features observed in well-known Li ion insertion systems such as lithium iron phosphate (LFP) and lithium cobalt oxide (LCO). Then we demonstrated the use of impedance spectroscopy on a post lithium ion system, specifically lithium-sulfur battery. We showed that the complex response of the sulfur cathode can be better understood if the usual porous electrode morphology is replaced with a flat electrode and a symmetrical cell instead of asymmetrical is used. Once the main transport-reaction mechanism has been identified, the flat electrode can be replaced by a porous in order to take into account the effect of porosity. There, one of the main issues is how to connect (couple) the response of a porous electrode with that of porous separator. We have managed to carry out this coupling by proposing an appropriate transmission line model. The latter can describe all the measured features of a porous carbon electrode in contact with polysulfides. Finally, we extended the same basic concept of transmission line for porous electrodes to a cycled lithium electrode. Namely, it appears that the dendrites formed during charge-discharge represent a conceptually similar framework for transmission line modeling as the porous carbon electrode with surface reactions (such as in the case of polysulfides). The proposed transmission line is able, for the first time, to quantitatively - and with physically sound elements - reproduce the response of dendritic lithium.

Acknowledgements: This work was financially support by Slovene Research Agency and through Helis project (European Union's Horizon 2020 research and innovation program under Grant Agreement No. 666221).

\section{References}

[1] M. Winter, R. J. Brodd, Chemical Reviews 104 (2004) 4245-4270.

[2] B. Scrosati, Journal of Solid State Electrochemistry 15 (2011) 1623-1630.

[3] M. Gaberscek, J. Moskon, B. Erjavec, R. Dominko, J. Jamnik, Electrochemical and Solid-State Letters 11 (2008) A170-A174.

[4] J.-M. Atebamba, J. Moskon, S. Pejovnik, and M. Gaberscek, Journal of the Electrochemical Society 157 (2010) A1218-A1228.

[5] M. Doyle, J. P. Meyers, and J. Newman Journal of the Electrochemical Society 147 (2000) 99-110.

[6] J. P. Meyers, M. Doyle, R. M. Darling, and J. Newman, Journal of the Electrochemical Society 147 (2000) 2930-2940.

[7] B. C. Han, A. Van der Ven, D. Morgan, and G. Ceder, Electrochimica Acta 49 (2004) 4691-4699.

[8] Y.-S. Su, A. Manthiram, Nature Communications 3 (2012) article number: 1166.

[9] X. Ji, K. T. Lee, L. F. Nazar, Nature Materials 8 (2009) 500-506.

[10] L. Wang, Y. Wang, Y. Xia, Energy \& Environmental Science 8 (2015) 1551-1558. 
[11] S. Drvaric Talian, J. Moskon, R. Dominko, M. Gaberscek, ACS Applied Materials \& Interfaces 9 (2017) 29760-29770.

[12] M. Adamic, S. Drvaric Talian, A.R. Sinigoj, I. Humar, J. Moskon, M. Gaberscek, Journal of the Electrochemical Society 166 (2019) A5045-A5053.

[13] S. Devan, V. R. Subramanian, R. E. White, Journal of the Electrochemical Society 151 (2004) A905A913.

[14] J. Huang, J. Zhang, Journal of the Electrochemical Society 163 (2016) A1983-A2000.

[15] S. Drvarič Talian, Jože Moškon, R. Dominko, M. Gaberšček, Electrochimica Acta 302 (2019) 169-179.

[16] J. Bisquert, Physical Chemistry Chemical Physics 2 (2000) 4185-4192.

[17] E. Peled, in Lithium Batteries, J. Gabano, Editor, Academic Press, 1983.

[18] E. Peled, Journal of the Electrochemical Society 126 (1979) 2047-2051.

[19] X.-B. Cheng, R. Zhang, C.-Z. Zhao, Q. Zhang, Chemical Reviews 117 (2017) 10403-10473.

[20] E. Peled, S. Menkin, Journal of the Electrochemical Society 164 (2017) A1703-A1719.

[21] D. Aurbach, Y. Ein-Ely, A. Zaban, Journal of the Electrochemical Society 141 (1994) L1-L3.

[22] D. Aurbach, E. Zinigrad, Y. Cohen, H. A. Teller, Solid State lonics 148 (2002) 405-416.

[23] S. Drvarič Talian, J. Bobnar, A. R. Sinigoj, I. Humar, M. Gaberšček, Journal of Physical Chemistry C, 123 (2019) 27997-28007.

(C)2020 by the authors; licensee IAPC, Zagreb, Croatia. This article is an open-access article distributed under the terms and conditions of the Creative Commons Attribution license (http://creativecommons. org/licenses/by/4.0/) 